\title{
Correction to: Dysplastic features seen in a patient with acute myeloid leukemia harboring the KMT2A-TET1 fusion gene
}

\author{
Hidetsugu Kawai ${ }^{1}\left[\right.$ [ Hiromichi Matsushita ${ }^{2}$ - Yasuyuki Aoyama ${ }^{3} \cdot$ Keiko Matsui $^{1} \cdot$ Makoto Onizuka $^{1}$. \\ Kiyoshi Ando ${ }^{1}$
}

Published online: 12 June 2018

(c) The Japanese Society of Hematology 2018

\section{Correction to: International Journal of Hematology https://doi.org/10.1007/s12185-018-2469-4}

In the original publication of the article, the title was incorrectly published as "Dysplastic features seen in a patient with acute myeloid leukemia harboring the KTM2A-TET1 fusion gene". The correct title should be "Dysplastic features seen in a patient with acute myeloid leukemia harboring the KMT2A-TET1 fusion gene".

The original article can be found online at https://doi.org/10.1007/ s12185-018-2469-4.

Hiromichi Matsushita hirommat@ncc.go.jp

1 Department of Hematology/Oncology, Tokai University School of Medicine, Isehara, Kanagawa, Japan

2 Division of Pathology and Clinical Laboratories, National Cancer Center Hospital, Chuo-ku, Tokyo, Japan

3 Department of Hematology, Japanese Red Cross Hadano Hospital, Hadano, Kanagawa, Japan 